\title{
INTERPOLATION OF MULTILINEAR OPERATORS ACTING BETWEEN QUASI-BANACH SPACES
}

\author{
L. GRAFAKOS, M. MASTYŁO, AND R. SZWEDEK
}

(Communicated by Alexander Iosevich)

\begin{abstract}
We show that interpolation of multilinear operators can be lifted to multilinear operators from spaces generated by the minimal methods to spaces generated by the maximal methods of interpolation defined on a class of couples of compatible $p$-Banach spaces. We also prove the multilinear interpolation theorem for operators on Calderón-Lozanovskii spaces between $L_{p}$-spaces with $0<p \leq 1$. As an application we obtain interpolation theorems for multilinear operators on quasi-Banach Orlicz spaces.
\end{abstract}

\section{INTRODUCTION}

In the study of the many problems which appear in various areas of analysis it is essential to know whether important operators are bounded between certain quasi-Banach spaces. Motivated in particular by applications in harmonic analysis, we are interested in proving new abstract multilinear interpolation theorems for multilinear operators between quasi-Banach spaces. Based on ideas from the theory of operators between Banach spaces, we use the universal method of interpolation defined on proper classes of quasi-Banach spaces. It should be pointed out that in general the interpolation methods used in the case of Banach spaces do not apply in the setting of quasi-Banach spaces. The main reason is that the topological dual spaces of quasi-Banach spaces could be trivial and the same may be true for spaces of continuous linear operators between spaces from a wide class of quasi-Banach spaces.

We introduce relevant notation and recall some definitions. Let $(X,\|\cdot\|)$ be a quasi-normed space. A quasi-norm induces locally bounded topology. A complete quasi-normed space is called quasi-Banach space. If in addition we have for some $0<p \leq 1$

$$
\|x+y\|^{p} \leq\|x\|^{p}+\|y\|^{p}, \quad x, y \in X,
$$

then $X$ is said to be a $p$-Banach space (or if $p=1$ a Banach space). A theorem of Aoki and Rolewicz (see [6]) states that every quasi-Banach space is $p$-normed for some $p \in(0,1]$.

Received by the editors August 16, 2012.

2010 Mathematics Subject Classification. Primary 46B70, 46M35.

Key words and phrases. Interpolation spaces, quasi-Banach spaces, multilinear operators, functor Calderón-Lozanovskii spaces, Orlicz spaces.

The first author was supported by the NSF grant DMS 0900946.

The second author was supported by the National Science Centre (NCN), Poland, grant No. 2011/01/B/ST1/06243. 
Throughout the paper we use the standard notion from Banach space theory and interpolation theory. We refer to [2] and [3] for the fundamentals of interpolation theory that will be of use.

A pair $\bar{A}=\left(A_{0}, A_{1}\right)$ of quasi-Banach ( $p$-Banach) spaces is called a quasi-Banach ( $p$-Banach) couple if $A_{0}$ and $A_{1}$ are both algebraically and topologically embedded in some Hausdorff topological vector space. For a quasi-Banach ( $p$-Banach) couple $\bar{A}=\left(A_{0}, A_{1}\right)$ we define quasi-Banach spaces $A_{0} \cap A_{1}$ and $A_{0}+A_{1}$ equipped with the natural norms. A quasi-Banach space $A$ is called intermediate with respect to $\bar{A}$ provided $A_{0} \cap A_{1} \hookrightarrow A \hookrightarrow A_{0}+A_{1}$, where $\hookrightarrow$ denotes the continuous inclusion map.

Let $\bar{A}=\left(A_{0}, A_{1}\right)$ and $\bar{B}=\left(B_{0}, B_{1}\right)$ be quasi-Banach couples. We denote by $\mathcal{L}(\bar{A}, \bar{B})$ the Banach space of all linear operators $T: A_{0}+A_{1} \rightarrow B_{0}+B_{1}$ such that the restrictions of $T$ to $A_{i}$ are bounded operators from $A_{i}$ to $B_{i}$ for $i=0,1$. We equip $\mathcal{L}(\bar{A}, \bar{B})$ with the quasi-norm

$$
\|T\|_{\bar{A} \rightarrow \bar{B}}=\max \left\{\|T\|_{A_{0} \rightarrow B_{0}},\|T\|_{A_{1} \rightarrow B_{1}}\right\} .
$$

Let $\bar{A}$ and $\bar{X}$ be quasi-Banach couples. Following Aronszajn and Gagliardo [1], the orbit of an element $a \in A_{0}+A_{1}$ in $\bar{X}$ is the quasi-Banach space $O_{\bar{A}}(a, \bar{X})=$ $\{T a ; T \in \mathcal{L}(\bar{A}, \bar{X})\}$ equipped with the norm

$$
\|x\|=\inf \left\{\|T\|_{\bar{A} \rightarrow \bar{X}} ; T a=x\right\} .
$$

If we assume that $A_{0}+A_{1}$ has a total dual space, then $X_{0} \cap X_{1} \hookrightarrow O_{\bar{A}}(a, \bar{X})$, and so $F(\cdot):=O_{\bar{A}}(a, \cdot)$ is an exact interpolation functor, i.e., for any quasi-Banach couples $\bar{X}$ and $\bar{Y}$ and every operator $T \in \mathcal{L}(\bar{X}, \bar{Y})$ we have $T: F(\bar{X}) \rightarrow F(\bar{Y})$ with

$$
\|T\|_{F(\bar{X}) \rightarrow F(\bar{Y})} \leq\|T\|_{\bar{X} \rightarrow \bar{Y}} .
$$

Fix $0<p \leq 1$. Let $\bar{A}=\left(A_{0}, A_{1}\right)$ be a quasi-Banach couple such that $A_{0}+A_{1}$ has a total dual space, and let $A$ be an intermediate quasi-Banach space with respect to $\bar{A}$. For any quasi-Banach couple $\bar{X}=\left(X_{0}, X_{1}\right)$ we define the $p$-interpolation orbit space $G_{\bar{A}, p}^{A}(\bar{X})$ as the space of all $x \in X_{0}+X_{1}$ such that

$$
\left.x=\sum_{n=1}^{\infty} T_{n} a_{n} \quad \text { (convergence in } X_{0}+X_{1}\right),
$$

where $T_{n} \in \mathcal{L}(\bar{A}, \bar{X}), a_{n} \in A$, and $\sum_{n=1}^{\infty}\left(\left\|T_{n}\right\|_{\bar{A} \rightarrow \bar{X}}\left\|a_{n}\right\|_{A}\right)^{p}<\infty$. We set

$$
\|x\|_{G_{p}}=\inf \left(\sum_{n=1}^{\infty}\left(\left\|T_{n}\right\|_{\bar{A} \rightarrow \bar{X}}\left\|a_{n}\right\|_{A}\right)^{p}\right)^{1 / p},
$$

where the infimum is taken over all admissible representations of $x$ as above.

We notice here that if $\bar{X}$ is a $p$-Banach couple, then $G_{\bar{A}, p}^{A}(\bar{X})$ is a $p$-Banach space, intermediate with respect to $\bar{X}$. Moreover, we have

$$
G_{\bar{A}, p}^{A}(\bar{X}) \hookrightarrow F(\bar{X})
$$

for any interpolation functor $F$ such that $F(\bar{X})$ is $p$-normed space and $A \hookrightarrow F(\bar{A})$ (see [8, Proposition 2.1]). 
Suppose we are given a quasi-Banach couple $\bar{B}$ and an intermediate quasi-Banach space $B$ with respect to the couple $\bar{B}$. Following [1, we define for any Banach couple $\bar{X}$ the space $H \frac{B}{B}(\bar{X})$ of all $x \in X_{0}+X_{1}$ such that $\sup _{\|T\|_{\bar{X} \rightarrow \bar{B}} \leq 1}\|T x\|_{B}<\infty$. The quasi-norm in $H_{\bar{B}}(\bar{X})$ is given by

$$
\|x\|_{H \frac{B}{B}(\bar{X})}=\sup \left\{\|T x\|_{B} ;\|T\|_{\bar{X} \rightarrow \bar{B}} \leq 1\right\} .
$$

Note that if $F$ is an interpolation method, then $F(\bar{X}) \hookrightarrow H_{B} B(\bar{X})$ for any quasiBanach couple provided $F(\bar{B}) \hookrightarrow B$. This property, according to Aronszajn and Gagliardo [1, motivates calling $H \frac{B}{B}$ the maximal interpolation functor.

\section{MAIN RESUlts}

For each $m \in \mathbb{N}$ the product $X_{1} \times \cdots \times X_{m}=\prod_{i=1}^{m} X_{i}$ of quasi-Banach spaces is equipped with the norm $\left\|\left(x_{1}, \ldots, x_{m}\right)\right\|=\max _{1 \leq i \leq m}\left\|x_{i}\right\|_{X_{i}}$. We denote by $\mathcal{L}_{m}\left(X_{1} \times \cdots \times X_{m}, Y\right)$ the quasi-Banach space of all $m$-linear bounded operators defined on $X_{1} \times \cdots \times X_{m}$ with values in a quasi-Banach space $Y$, equipped with the quasi-norm

$$
\|T\|=\sup \left\{\left\|T\left(x_{1}, \ldots, x_{m}\right)\right\|_{Y} ;\left\|x_{1}\right\|_{X_{1}} \leq 1, \ldots,\left\|x_{m}\right\|_{X_{m}} \leq 1\right\} .
$$

As in the case where $m=1$, we write $\mathcal{L}\left(X_{1}, Y\right)$ instead of $\mathcal{L}_{1}\left(X_{1}, Y\right)$.

Let $\bar{Y}=\left(Y_{0}, Y_{1}\right)$ and $\bar{X}_{i}=\left(X_{0 i}, X_{1 i}\right)$ for each $1 \leq i \leq m$ be couples of quasiBanach spaces. If an operator $T \in \mathcal{L}_{m}\left(\prod_{i=1}^{m}\left(X_{0 i}+X_{1 i}\right), Y_{0}+Y_{1}\right)$ is such that the restriction of $T$ is bounded from $X_{j 1} \times \cdots \times X_{j m}$ to $Y_{j}$ for $j=0,1$, then we write $T \in \mathcal{L}_{m}\left(\prod_{i=1}^{m} \bar{X}_{i}, \bar{Y}\right)$.

Assume that $X_{i}$ are quasi-Banach spaces intermediate with respect to $\bar{X}_{i}$ for $1 \leq i \leq m$ and $Y$ is a quasi-Banach intermediate with respect to $\bar{Y}$. If there exists a finite constant $C>0$ such that for every $T \in \mathcal{L}_{m}\left(\prod_{i=1}^{m} \bar{X}_{i}, \bar{Y}\right)$ the restriction of $T$ is bounded from $X_{1} \times \cdots \times X_{m}$ to $Y$ with $\|T\| \leq C$, then $X_{1}, \ldots, X_{m}$ and $Y$ are called $C$-multilinear interpolation spaces with respect to $\left(\bar{X}_{1}, \ldots, \bar{X}_{m}\right)$ and $\bar{Y}$ (we write for short $\left(X_{1}, \ldots, X_{m} ; Y\right) \in \mathcal{M i n t}_{C}\left(\bar{X}_{1}, \ldots, \bar{X}_{m} ; \bar{Y}\right)$ and $\left(X_{1}, X_{2} ; Y\right) \in \operatorname{int}_{C}\left(\bar{X}_{1}, \bar{X}_{2}\right)$ in the case where $m=1)$.

Our first result is the following.

Theorem 2.1. Let $G_{\bar{A}_{i}, p}^{A_{i}}$ be a p-interpolation orbit for each $1 \leq i \leq m$ and let $H \frac{B}{B}$ be a maximal interpolation method. Assume that $\left(A_{1}, \ldots, A_{m} ; B\right) \in \operatorname{Mint}_{C}\left(\bar{A}_{1}, \ldots\right.$, $\left.\bar{A}_{m} ; \bar{B}\right)$. Then for any $p$-Banach couples $\bar{X}_{1}, \ldots, \bar{X}_{m}$ and any quasi-Banach couple $\bar{Y}$, we have

$$
\left(G_{\bar{A}_{1}, p}^{A_{1}}\left(\bar{X}_{1}\right), \ldots, G_{\bar{A}_{m}, p}^{A_{m}}\left(\bar{X}_{m}\right) ; H \frac{B}{B}(\bar{Y})\right) \in \mathcal{M i n t}_{C}\left(\bar{X}_{1}, \ldots, \bar{X}_{m} ; \bar{Y}\right) .
$$

Proof. Let $X_{i}:=G_{\bar{A}_{i}, p}^{A_{i}}\left(\bar{X}_{i}\right)$ for each $1 \leq i \leq m$ and $Y:=H_{\bar{B}}(\bar{Y})$. For $j=0,1$ fix $T \in \mathcal{L}_{m}\left(\prod_{i=1}^{m}\left(X_{0 i}+X_{1 i}\right), Y_{0}+Y_{1}\right)$ such that $\|T\|_{\mathcal{L}_{m}\left(X_{j 1}, \ldots, X_{j n} ; Y_{j}\right)} \leq 1$. Assume that $x_{i} \in X_{i}$ and $x_{i}=S_{i} a_{i}$ with $a_{i} \in A_{i}$, where $S_{i}: \bar{A}_{i} \rightarrow \bar{X}_{i}$. For a given $R: \bar{Y} \rightarrow \bar{B}$ with $\|R\|_{\bar{Y} \rightarrow \bar{B}} \leq 1$ define an operator $U_{R}: \prod_{i=1}^{m}\left(A_{0 i}+A_{1 i}\right) \rightarrow B_{0}+B_{1}$ by setting

$$
U_{R}\left(v_{1}, \ldots, v_{m}\right)=R T\left(S_{1} v_{1}, \ldots, S_{m} v_{m}\right), \quad\left(v_{1}, \ldots, v_{m}\right) \in \prod_{i=1}^{m}\left(A_{0 i}+A_{1 i}\right) .
$$


For $j=0,1$ we have

$$
\left\|U_{R}\left(u_{1}, \ldots, u_{m}\right)\right\|_{B_{j}} \leq\|R\|_{\bar{Y} \rightarrow \bar{B}}\|T\|_{\mathcal{L}_{m}\left(X_{j 1}, \ldots, X_{j n} ; Y_{j}\right)} \prod_{i=1}^{m}\left\|S_{i}\right\|_{\bar{A}_{i} \rightarrow \bar{X}_{i}}\left\|v_{i}\right\|_{A_{j i}},
$$

and so $U_{R} \in \mathcal{L}_{m}\left(\bar{A}_{1} \times \cdots \times A_{m}, \bar{B}\right)$ and its norm satisfies

$$
\left\|U_{R}\right\| \leq \prod_{i=1}^{m}\left\|S_{i}\right\|_{\bar{A}_{i} \rightarrow \bar{X}_{i}} .
$$

Our hypothesis gives that $U_{R} \in \mathcal{L}_{m}\left(A_{1}, \ldots, A_{m}, B\right)$ and

$$
\left\|U_{R}\right\|_{\mathcal{L}_{m}\left(A_{1}, \ldots, A_{m}, B\right)} \leq C \prod_{i=1}^{m}\left\|S_{i}\right\|_{\bar{A}_{i} \rightarrow \bar{X}_{i}} .
$$

Consequently, we obtain

$$
\begin{aligned}
\left\|T\left(x_{1}, \ldots, x_{m}\right)\right\|_{Y} & \leq \sup \left\{\left\|R T\left(S_{1} v_{1}, \ldots, S_{m} v_{m}\right)\right\|_{B} ;\|R\|_{\bar{Y} \rightarrow \bar{B}} \leq 1\right\} \\
& =\sup \left\{\left\|U_{R}\left(a_{1}, \ldots, a_{m}\right)\right\|_{B} ;\|R\|_{\bar{Y} \rightarrow \bar{B}} \leq 1\right\} \\
& \leq C \prod_{i=1}^{m}\left\|S_{i}\right\|_{\bar{A}_{i} \rightarrow \bar{X}_{i}}\left\|a_{i}\right\|_{A_{i}} .
\end{aligned}
$$

Suppose now that for each $1 \leq i \leq m$

$$
\left.x_{i}=\sum_{j=1}^{\infty} S_{i j} a_{i j} \quad \text { (convergence in } X_{0 i}+X_{1 i}\right),
$$

where $S_{i j}: \bar{A}_{i} \rightarrow \bar{X}_{i}, a_{i j} \in A_{i}$, are such that

$$
\sum_{j=1}^{\infty}\left(\left\|S_{i j}\right\|_{\bar{A}_{j} \rightarrow \bar{X}_{j}}\left\|a_{i j}\right\|_{A_{i}}\right)^{p}<\infty
$$

Since $T \in \mathcal{L}_{m}\left(\prod_{i=1}^{m}\left(X_{0 i}+X_{1 i}\right), Y_{0}+Y_{1}\right)$ we conclude that

$$
\left.T\left(x_{1}, \ldots, x_{m}\right)=\sum_{j_{1}=1}^{\infty} \cdots \sum_{j_{m}=1}^{\infty} T\left(S_{1 j_{1}} a_{1 j_{1}}, \ldots, S_{m j_{m}} a_{m j_{m}}\right) \quad \text { (convergence in } Y_{0}+Y_{1}\right) \text {. }
$$

Estimate (11) yields for each $j_{1}, \ldots, j_{m}$

$$
\left\|T\left(S_{1 j_{1}} a_{1 j_{1}}, \ldots, S_{m j_{m}} a_{m j_{m}}\right)\right\|_{Y} \leq C \prod_{i=1}^{m}\left\|S_{i j_{i}}\right\|_{\bar{A}_{j_{i}} \rightarrow \bar{X}_{j_{i}}}\left\|a_{j_{i}}\right\|_{A_{j_{i}}},
$$

and so

$$
\begin{aligned}
\left\|T\left(x_{1}, \ldots, x_{m}\right)\right\|_{Y} & \leq C\left(\sum_{j_{1}=1}^{\infty} \cdots \sum_{j_{m}=1}^{\infty}\left\|T\left(S_{1 j_{1}} a_{1 j_{1}}, \ldots, S_{m j_{m}} a_{m j_{m}}\right)\right\|_{Y}^{p}\right)^{1 / p} \\
& \leq C\left(\sum_{j_{1}=1}^{\infty} \cdots \sum_{j_{m}=1}^{\infty} \prod_{i=1}^{m}\left(\left\|S_{i j_{i}}\right\|_{\bar{A}_{i} \rightarrow \bar{X}_{i}}\left\|a_{i j}\right\|_{A_{i}}\right)^{p}\right)^{1 / p} \\
& =C \prod_{i=1}^{m}\left(\sum_{j=1}^{\infty}\left(\left\|S_{i j}\right\|_{\bar{A}_{j_{i}} \rightarrow \bar{X}_{j}}\left\|a_{i j}\right\|_{A_{i}}\right)^{p}\right)^{1 / p} .
\end{aligned}
$$

Combining the above estimates, we conclude that $T \in \mathcal{L}_{m}\left(X_{1} \times \cdots \times X_{m}, Y\right)$ with $\|T\| \leq C$, and this completes the proof. 
Our results could be applied to the real methods of interpolation. Let $0<p \leq 1$. Following [9, a quasi-Banach space $E$ is said to be $(p, J)$-non-trivial if

$$
E \hookrightarrow \ell_{p}+\ell_{p}\left(2^{-n}\right)
$$

Let $E$ be a non-trivial $(1, J)$ quasi-Banach lattice. For any quasi-Banach couple $\left(X_{0}, X_{1}\right)$ we denote by $J_{E}(\bar{X})$ the space of all $x \in X_{0}+X_{1}$, which can be represented in the form

$$
\left.x=\sum_{n=-\infty}^{\infty} x_{n}, \quad x_{n} \in X_{0} \cap X_{1} \quad \text { (convergence in } X_{0}+X_{1}\right),
$$

with $\left\{J\left(2^{n}, x_{n} ; \bar{X}\right)\right\} \in E$ where $J(t, x ; \bar{X})=\max \left\{\|x\|_{X_{0}}, t\|x\|_{X_{1}}\right\}$ for all $x \in X_{0} \cap$ $X_{1}, t>0$. The space $J_{E}(\bar{X})$ is said to be a $J$-space provided it is a quasi-Banach space under the quasi-norm,

$$
\|x\|=\inf \left\|\left\{J\left(2^{n}, x_{n} ; \bar{X}\right)\right\}\right\|_{E},
$$

where the infimum is taken over all representations of $x=\sum_{n} x_{n}$ as above.

Theorem 2.2. For each $1 \leq i \leq m$ let $\bar{X}_{i}$ be p-Banach couples and let $J_{E_{i}}\left(\bar{X}_{i}\right)$ be $J$-spaces generated by quasi-Banach lattices on $\mathbb{Z}$ intermediate between $\bar{\ell}_{p}=$ $\left(\ell_{p}, \ell_{p}\left(2^{-n}\right)\right)$, and let $H \frac{B}{B}$ be a maximal interpolation method. Assume that $\left(E_{1}, \ldots\right.$, $\left.E_{m} ; B\right) \in \mathcal{M i n t}_{C}\left(\bar{\ell}_{p}, \ldots, \bar{\ell}_{p} ; \bar{B}\right)$. Then for any quasi-Banach couple $\bar{Y}$ we have

$$
\left(J_{E_{1}}\left(\bar{X}_{1}\right), \ldots, J_{E_{m}}\left(\bar{X}_{m}\right) ; H \frac{B}{B}(\bar{Y})\right) \in \mathcal{M i n t}_{C}\left(\bar{X}_{1}, \ldots, \bar{X}_{m} ; \bar{Y}\right) .
$$

Proof. It follows from [8, Theorem 3.2] that the continuous inclusion map

$$
J_{E_{i}}\left(\bar{X}_{i}\right) \hookrightarrow G_{\bar{\ell}_{p}, p}^{E_{i}}\left(\bar{X}_{i}\right), \quad 1 \leq i \leq m,
$$

has norm less than or equal to 1 . Thus the required statement follows from Theorem 2.1

We refer to [5] where interpolation of bilinear operators between quasi-Banach spaces was studied by the method of means.

\section{Multilinear interpolation Between Orlicz SPACES}

In what follows we let $(\Omega, \mu):=(\Omega, \Sigma, \mu)$ be a complete $\sigma$-finite measure space and let $L^{0}(\Omega, \mu)=L^{0}(\mu)$ denote the space of equivalence classes of real valued measurable functions on $\Omega$, equipped with the topology of convergence (in the measure $\mu$ ) on sets of finite measure. By a quasi-Banach lattice on $\Omega$ we mean a quasi-Banach space $X$ which is a subspace of $L^{0}(\mu)$ such that there exists $u \in X$ with $u>0$ and if $|f| \leq|g|$ a.e., where $g \in X$ and $f \in L^{0}(\mu)$, then $f \in X$ and $\|f\|_{X} \leq\|g\|_{X}$. A quasi-Banach lattice $X$ is said to be maximal if its unit ball $B_{X}=\{x ;\|x\| \leq 1\}$ is a closed subset in $L^{0}(\mu)$.

In the special case when $\Omega=\mathbb{Z}$ is the set of integers and $\mu$ is the counting measure, then a quasi-Banach lattice $E$ on $\Omega$ is called a quasi-Banach sequence space on $\mathbb{Z}$.

If $X$ is a quasi-Banach lattice on $(\Omega, \mu)$ and $w \in L^{0}(\mu)$ with $w>0$ a.e., we define the weighted quasi-Banach lattice $X(w)$ by $\|x\|_{X(w)}=\|x w\|_{X}$.

Throughout the rest of the paper for given measure spaces $\left(\Omega_{i}, \Sigma_{i}, \mu_{i}\right), 1 \leq$ $i \leq m$, we let $(\Omega, \Sigma, \mu)$ be a product measure space with $\Omega:=\Omega_{1} \times \cdots \times \Omega_{m}$, $\Sigma:=\Sigma_{1} \times \cdots \times \Sigma_{m}$ and $\mu:=\mu_{1} \times \cdots \times \mu_{m}$ be a product measure space. 
We define a map $\otimes: L^{0}\left(\mu_{1}\right) \times \cdots \times L^{0}\left(\mu_{m}\right) \rightarrow L^{0}(\mu)$ by

$$
\otimes\left(f_{1}, \ldots, f_{m}\right)=f_{1} \otimes \cdots \otimes f_{m}, \quad\left(f_{1}, \ldots, f_{m}\right) \in L^{0}\left(\mu_{1}\right) \times \cdots \times L^{0}\left(\mu_{m}\right),
$$

where $f_{1} \otimes \cdots \otimes f_{m}\left(\omega_{1}, \ldots, \omega_{m}\right)=f_{1}\left(\omega_{1}\right) \cdots f_{m}\left(\omega_{m}\right)$ for all $\left(\omega_{1}, \ldots, \omega_{m}\right) \in$ $\Omega_{1} \times \cdots \times \Omega_{m}$.

Next we state a useful result in terms of applications.

Theorem 3.1. Let $0<p_{0}, p_{1} \leq 1$ and $X_{i}$ be quasi-Banach lattices on $\left(\Omega_{i}, \mu_{i}\right)$ that are intermediate with respect to $\left(L^{p_{0}}\left(\mu_{i}\right), L^{p_{1}}\left(\mu_{i}\right)\right)$ for $1 \leq i \leq m$ and let $Y$ be a quasi-Banach space intermediate with respect to a quasi-Banach couple $\left(Y_{0}, Y_{1}\right)$. Assume $X$ is a quasi-Banach function lattice on $(\Omega, \mu)$ such that

$$
\bigotimes: X_{1} \times \cdots \times X_{m} \rightarrow X
$$

with $\|\otimes\| \leq C_{1}$ and $(X, Y) \in$ int $_{C_{2}}\left(\left(L^{p_{0}}(\mu), L^{p_{1}}(\mu)\right) ;\left(Y_{0}, Y_{1}\right)\right)$. Then, with $C=C_{1} C_{2}$, we have

$\left(X_{1}, \ldots, X_{m} ; X\right) \in \mathcal{M i n t}_{C}\left(\left(L^{p_{0}}\left(\mu_{1}\right), L^{p_{1}}\left(\mu_{1}\right)\right) \times \cdots \times\left(L^{p_{0}}\left(\mu_{m}\right), L^{p_{1}}\left(\mu_{m}\right)\right) ;\left(Y_{0}, Y_{1}\right)\right)$.

Proof. In [13] Vogt identifies the tensor product

$$
L^{p}\left(\mu_{1}\right) \widehat{\otimes}_{p} \cdots \widehat{\otimes}_{p} L^{p}\left(\mu_{m}\right)
$$

for $m=2$; however, the proof works for each positive integer $m \geq 2$. In our setting this implies that there are continuous linear operators $T_{0}: L^{p_{0}}(\mu) \rightarrow Y_{0}$ and $T_{1}: L^{p_{1}}(\mu) \rightarrow Y_{1}$ such that

$$
T\left(f_{1}, \ldots, f_{m}\right)=T_{0} \otimes\left(f_{1}, \ldots, f_{m}\right), \quad\left(f_{1}, \ldots, f_{m}\right) \in \prod_{i=1}^{m} L^{p_{0}}\left(\mu_{i}\right)
$$

and

$$
T\left(g_{1}, \ldots, g_{m}\right)=T_{1} \otimes\left(g_{1}, \ldots, g_{m}\right), \quad\left(g_{1}, \ldots, g_{m}\right) \in \prod_{j=1}^{m} L^{p_{1}}\left(\mu_{i}\right) .
$$

In particular this yields that for all $\left(f_{1}, \ldots, f_{m}\right) \in \prod_{i=1}^{m}\left(L_{p_{0}}\left(\mu_{i}\right) \cap L^{p_{1}}\left(\mu_{i}\right)\right)$,

$$
T\left(f_{1}, \ldots, f_{m}\right)=T_{0}\left(f_{1} \otimes \cdots \otimes f_{m}\right)=T_{1}\left(f_{1} \otimes \cdots \otimes f_{m}\right) .
$$

The density argument (via (2) ) implies that $T_{0}=T_{1}$ on $L^{p_{0}}(\mu) \cap L^{p_{1}}(\mu)$. Consequently, the operator $\widetilde{T}: L^{p_{0}}(\mu)+L^{p_{1}}(\mu) \rightarrow Y_{0}+Y_{1}$ given by $\widetilde{T}(f)=T_{0}\left(f_{0}\right)+T_{1}\left(f_{1}\right)$ for any $f=f_{0}+f_{1}$ with $f_{j} \in L_{p_{j}}(\mu)$ for $j=0,1$ is well defined. Since $\widetilde{T}=T_{j}$ on $L^{p_{j}}(\mu)$, it follows that

$$
\widetilde{T}:\left(L^{p_{0}}(\mu), L^{p_{1}}(\mu)\right) \rightarrow\left(Y_{0}, Y_{1}\right) .
$$

Hence we have $T=\widetilde{T} \otimes$ with

$T:\left(L^{p_{0}}\left(\mu_{1}\right), L^{p_{1}}\left(\mu_{1}\right)\right) \times \cdots \times\left(L^{p_{m}}\left(\mu_{m}\right), L_{p_{m}}\left(\mu_{m}\right)\right) \stackrel{\otimes}{\longrightarrow}\left(L^{p_{0}}(\mu), L^{p_{1}}(\mu)\right) \stackrel{\widetilde{T}}{\longrightarrow}\left(Y_{0}, Y_{1}\right)$.

Our interpolation hypotheses imply that

$$
T: X_{1} \times \cdots \times X_{m} \stackrel{\otimes}{\longrightarrow} X \stackrel{\widetilde{T}}{\longrightarrow} Y
$$

with $\|T\| \leq\|\widetilde{T}\|\|\otimes\| \leq C_{1} C_{2}$, and this completes the proof. 
To obtain a variety of applications we recall some interpolation constructions. Let $\Phi$ denote the set of non-vanishing functions $\varphi:[0, \infty) \times[0, \infty) \rightarrow[0, \infty)$, which are non-decreasing in each variable and positively homogeneous of degree one. We define $\Phi_{0}$ to be a subset of all $\varphi \in \Phi$ such that $\varphi(1, t) \rightarrow 0$ and $\varphi(t, 1) \rightarrow 0$ as $t \rightarrow 0$.

We consider the Banach couples $\bar{c}_{0}=\left(c_{0}, c_{0}\left(2^{-n}\right)\right)$ and $\bar{\ell}_{\infty}:=\left(\ell_{\infty}, \ell_{\infty}\left(2^{-n}\right)\right)$ of sequences on $\mathbb{Z}$. If $\varphi \in \Phi$ (resp., $\varphi \in \Phi_{0}$ ), then $a_{\varphi} \in c_{0}+c_{0}\left(2^{-n}\right)$ (resp., $\left.a_{\varphi} \in \ell_{\infty}+\ell_{\infty}\left(2^{-n}\right)\right)$.

For any $\varphi \in \Phi$ (resp., $\varphi \in \Phi_{0}$ ) and any quasi-Banach couple $\bar{X}$, we denote by $\left\langle X_{0}, X_{1}\right\rangle_{\varphi}$ (resp., $\varphi_{\ell}(\bar{X})$ ) the interpolation orbit space $O_{\bar{c}_{0}}\left(a_{\varphi}, \bar{X}\right)$ (resp., $\left.O_{\bar{\ell}_{\infty}}\left(a_{\varphi}, \bar{X}\right)\right)$. It was shown in [10], [9] that for a large class of couples of quasiBanach lattices these spaces are connected with the Calderón-Lozanovskii spaces.

We recall that if $\bar{X}=\left(X_{0}, X_{1}\right)$ is a couple of quasi-Banach lattices on a measure space $(\Omega, \mu)$ and $\varphi \in \Phi$, then the Calderón-Lozanovskii space $\varphi(\bar{X})=\varphi\left(X_{0}, X_{1}\right)$ consists of all $x \in L^{0}(\mu)$ such that $|x|=\varphi\left(\left|x_{0}\right|,\left|x_{1}\right|\right) \mu$-a.e. for some $x_{j} \in X_{j}$ with $\left\|x_{j}\right\|_{X_{j}} \leq 1, j=0,1$. The space $\varphi(\bar{X})$ is a quasi-Banach lattice equipped with the quasi-norm $([7])$

$$
\|x\|_{\varphi(\bar{X})}:=\inf \left\{\max _{j=0,1}\left\|x_{j}\right\|_{X_{j}} ; \varphi\left(\left|x_{0}\right|,\left|x_{1}\right|\right)=|x|\right\} .
$$

If $\varphi$ is a concave function, then $\varphi\left(X_{0}, X_{1}\right)$ is a Banach lattice provided $\left(X_{0}, X_{1}\right)$ is a Banach couple (see [7]). In the case when $\varphi(s, t)=s^{1-\theta} t^{\theta}, \varphi(\bar{X})$ is the Calderón space (see [4]).

We note that in analogy with the Banach case the continuous inclusion

$$
\varphi\left(X_{0}, X_{1}\right) \hookrightarrow \varphi_{\ell}\left(X_{0}, X_{1}\right)
$$

holds for all couples $\left(X_{0}, X_{1}\right)$ of quasi-Banach lattices and $\varphi \in \Phi$. It was shown in [10, 11] (see also 9]) that for any $\varphi \in \Phi_{0}$ the following continuous inclusions hold for a large class $\mathcal{C}$ of couples $\left(X_{0}, X_{1}\right)$ of quasi-Banach lattices:

$$
\varphi_{l}\left(X_{0}, X_{1}\right) \hookrightarrow\left\langle X_{0}, X_{1}\right\rangle_{\varphi} \hookrightarrow \varphi_{\ell}\left(X_{0}, X_{1}\right)^{c},
$$

where $\varphi\left(X_{0}, X_{1}\right)$ is a Calderón-Lozanovskii space and $\varphi_{\ell}\left(X_{0}, X_{1}\right)^{c}$ is the Gagliardo completion of $\varphi\left(X_{0}, X_{1}\right)$ with respect $X_{0}+X_{1}$, i.e., the space of all limits in $X_{0}+X_{1}$ of sequences $\left\{x_{n}\right\}$ that are bounded in $X=\varphi\left(X_{0}, X_{1}\right)$. This space is equipped with the quasi-norm

$$
\|x\|_{X^{c}}=\inf _{\left\{x_{n}\right\}} \sup _{n \geq 1}\left\|x_{n}\right\|_{X}
$$

where $\left\{x_{n}\right\} \subset X$ has the same meaning as above.

The class $\mathcal{C}$ contains $p$-convex quasi-Banach lattices with $1 \leq p<\infty$. Recall that a quasi-Banach lattice $X$ is called $p$-convex if there exists a constant $C>0$ such that if $x_{1}, \ldots, x_{n} \in X$, then

$$
\left\|\left(\sum_{i=1}^{n}\left|x_{i}\right|^{p}\right)^{1 / p}\right\|_{X} \leq C\left(\sum_{i=1}^{n}\left\|x_{i}\right\|_{X}^{p}\right)^{1 / p} .
$$

Notice that if $\varphi \in \Phi$, then $\varphi=\varphi_{0}+\varphi_{1}$, where $\varphi_{1}(s, t):=a s+b t$ for all $s, t \geq 0$ with $a=\lim _{s \rightarrow 0+} \varphi(1, s), b=\lim _{s \rightarrow 0+} \varphi(s, 1)$. Thus $a_{\varphi}=a_{\varphi_{0}}+a_{\varphi_{1}}$. Clearly

$$
\varphi(\bar{X})=\varphi_{0}(\bar{X})+\varphi_{1}(\bar{X})
$$

for any couple $\bar{X}$ of quasi-Banach lattices. Since $\varphi_{0} \in \Phi$, by combining the above remarks and the mentioned inclusions (3), we can easily deduce that the 
continuous inclusions

$$
\varphi\left(X_{0}, X_{1}\right) \hookrightarrow \varphi_{\ell}\left(X_{0}, X_{1}\right) \hookrightarrow \varphi\left(X_{0}, X_{1}\right)^{c}
$$

are true for every $\varphi \in \Phi$ and all quasi-Banach couples $\left(X_{0}, X_{1}\right) \in \mathcal{C}$.

Theorem 3.2. Let $L_{w_{0 i}}^{p_{0}}$ and $L_{w_{1 i}}^{p_{1}}$ with $0<p_{0}, p_{1} \leq 1$ be weighted spaces on measure spaces $\left(\Omega_{i}, \mu_{i}\right)$ for $1 \leq i \leq m$, and let $\left(Y_{0}, Y_{1}\right)$ be a couple of quasi-Banach spaces such that $Y_{0}$ is $p_{0}$-normed and $Y_{1}$ is a $p_{1}$-normed space. Assume that for some $\varphi_{1}, \ldots, \varphi_{m}, \varphi \in \Phi$ there exists $C>0$ such that $\varphi\left(1, t_{1} \cdots t_{m}\right) \leq C \varphi_{1}\left(1, t_{1}\right)$ $\cdots \varphi_{m}\left(1, t_{m}\right)$ for all $t_{1}, \ldots, t_{m} \geq 0$. Then any operator in

$$
\mathcal{L}_{m}\left(\left(L^{p_{0}}\left(\mu_{1}\right), L^{p_{1}}\left(\mu_{1}\right)\right) \times \cdots \times\left(L^{p_{0}}\left(\mu_{m}\right), L^{p_{1}}\left(\mu_{m}\right)\right),\left(Y_{0}, Y_{1}\right)\right)
$$

lies in

$$
\mathcal{L}_{m}\left(\varphi_{1}\left(L^{p_{0}}\left(\mu_{1}\right), L^{p_{1}}\left(\mu_{1}\right)\right) \times \cdots \times \varphi_{m}\left(L_{p_{0}}\left(\mu_{m}\right), L_{p_{1}}\left(\mu_{m}\right)\right), \varphi_{\ell}\left(Y_{0}, Y_{1}\right)\right) .
$$

Proof. It is clear that the condition $\varphi\left(1, t_{1} \cdots t_{m}\right) \leq C \varphi_{1}\left(1, t_{1}\right) \cdots \varphi_{m}\left(1, t_{m}\right)$ for all $t_{1}, \ldots, t_{m} \geq 0$ is equivalent to

$$
\varphi\left(s_{1} \cdots s_{m}, t_{1} \cdots t_{m}\right) \leq C \varphi_{1}\left(s_{1}, t_{1}\right) \cdots \varphi_{m}\left(s_{m}, t_{m}\right), \quad s_{i}, t_{i}>0, \quad 1 \leq i \leq m .
$$

This easily implies that for any measure space $\left(\Omega_{i}, \nu_{i}\right)$ with $1 \leq i \leq m$ and $\nu:=\nu_{1} \times \cdots \times \nu_{m}$,

$$
\bigotimes: \varphi_{1}\left(L^{p_{0}}\left(\nu_{1}\right), L^{p_{1}}\left(\nu_{1}\right)\right) \times \cdots \times \varphi_{m}\left(L^{p_{0}}\left(\nu_{m}\right), L^{p_{1}}\left(\nu_{m}\right)\right) \rightarrow \varphi\left(L^{p_{0}}(\nu), L^{p_{1}}(\nu)\right)
$$

with $\|\otimes\| \leq C$.

Without loss of generality we may assume that $1 / q:=1 / p_{0}-1 / p_{1}>0$. Based on ideas of Stein and Weiss [12, for each $1 \leq i \leq m$ set $\tau_{i}=\left(w_{1 i}^{1 / p_{0}} w_{0 i}^{1 / p_{1}}\right), \sigma_{i}=$ $\left(w_{0 i} / \tau_{i}\right)^{p_{0}}$ and $d \nu_{i}=\sigma_{i} d \mu_{i}$. For $f \in L^{0}\left(\nu_{i}\right)$ define $S_{i}(f)=\tau_{i} f$ and note that for each $1 \leq i \leq m$ and $j=0,1$ we have

$$
\left\|S_{i} f\right\|_{L^{p_{j}\left(\nu_{i}\right)}}=\|f\|_{L_{w_{j i}}^{p_{j}}}, \quad f \in L_{w_{j i}}^{p_{j}} .
$$

This shows that $S_{i}$ is a positive isometrical isomorphism between quasi-Banach couples $\left(L_{w_{0 i}}^{p_{0}}, L_{w_{1 i}}^{p_{1}}\right)$ and $\left(L^{p_{0}}\left(\nu_{i}\right), L^{p_{1}}\left(\nu_{i}\right)\right)$ for each $1 \leq i \leq m$, and so

$$
S_{i}\left(\varphi_{i}\left(L_{w_{0 i}}^{p_{0}}, L_{w_{1 i}}^{p_{1}}\right)\right)=\varphi_{i}\left(L^{p_{0}}\left(\nu_{i}\right), L^{p_{1}}\left(\nu_{i}\right)\right), \quad 1 \leq i \leq m .
$$

This implies that the operator $S$ given by

$$
S\left(f_{1}, \ldots, f_{m}\right):=T\left(S_{1}^{-1} f_{1}, \ldots, S_{m}^{-1} f_{m}\right)
$$

for all $\left(f_{1}, \ldots, f_{m}\right) \in \prod_{i=1}^{m}\left(L^{p_{0}}\left(\nu_{i}\right)+L^{p_{1}}\left(\nu_{i}\right)\right)$ satisfies

$$
S \in \mathcal{L}_{m}\left(\left(L^{p_{0}}\left(\nu_{0}\right), L^{p_{1}}\left(\nu_{0}\right)\right) \times \cdots \times\left(\left(L^{p_{0}}\left(\nu_{m}\right), L^{p_{1}}\left(\nu_{m}\right)\right),\left(Y_{0}, Y_{1}\right)\right) .\right.
$$

Applying Theorem 3.1, we conclude that $S$ is bounded from $\prod_{i=1}^{m} \varphi_{i}\left(L^{p_{i}}\left(\nu_{i}\right), L^{p_{1}}\left(\nu_{i}\right)\right)$ to $\varphi_{\ell}\left(Y_{0}, Y_{1}\right)$. The above isometries yield that the operator

$$
T: \varphi_{1}\left(L^{p_{0}}\left(\nu_{0}\right), L^{p_{1}}\left(\nu_{0}\right)\right) \times \cdots \times \varphi_{m}\left(L^{p_{0}}\left(\nu_{m}\right), L^{p_{1}}\left(\nu_{m}\right)\right) \rightarrow \varphi_{\ell}\left(Y_{0}, Y_{1}\right)
$$

is bounded, and this completes the proof. 
We give below applications of Theorem 3.2 to quasi-Banach Orlicz spaces. First we recall that if $\psi$ is an Orlicz function (i.e., $\psi:[0, \infty) \rightarrow[0, \infty)$ is an increasing, continuous function such that $\psi(0)=0$ ), then the Orlicz space $L_{\psi}$ on a given measure space $(\Omega, \mu)$ is defined to be a subspace of $L^{0}(\mu)$ consisting of all $f \in L^{0}(\mu)$ such that for some $\lambda>0$ we have $\int_{\Omega} \psi(\lambda|f|) d \mu<\infty$. We set

$$
\|f\|_{\psi}=\inf \left\{\lambda>0 ; \int_{\Omega} \psi(|f| / \lambda) d \mu \leq 1\right\} .
$$

If there exists $C>0$ such that $\psi(t / C) \leq \psi(t) / 2$ for all $t>0$, then

$$
\|f+g\|_{\psi} \leq C\left(\|f\|_{\psi}+\|g\|_{\psi}\right), \quad f, g \in L_{\psi},
$$

and so $L_{\psi}$ is a quasi-Banach space. In what follows we consider only Orlicz spaces $L^{\psi}$ generated by Orlicz functions $\psi$ which satisfy the above inequality.

It is well known (see [9, [11, pp. 460-461]) that for any $\varphi \in \Phi$ and any couple $\left(L_{w_{0}}^{p_{0}}, L_{w_{1}}^{p_{1}}\right)$ on a measure space $(\Omega, \mu)$ with $0<p_{0}, p_{1} \leq \infty$, we have

$$
\varphi\left(L_{w_{0}}^{p_{0}}, L_{w_{1}}^{p_{1}}\right)=L_{M}
$$

with equivalence of the quasi-norms, where $L_{M}$ is the generalized Orlicz space generated by the function $M(u, t)=\psi\left(\left(w_{1}(t)^{1 / p_{1}} w_{0}(t)^{-1 / p_{0}}\right)^{q}\right)\left(w_{0}(t) / w_{1}(t)\right)^{q}$ for all $u \geq 0$ and $t \in \Omega$. Here $1 / q=1 / p_{0}-1 / p_{1}$ and $\psi$ is an Orlicz function given by $\psi^{-1}(t)=\varphi\left(t^{1 / p_{0}}, t^{1 / p_{1}}\right)$ for all $t>0 . L_{M}$ is equipped with the quasi-norm

$$
\|f\|_{\Phi}=\inf \left\{\lambda>0 ; \int_{\Omega} M(|f(t)| / \lambda, t) d \mu \leq 1\right\} .
$$

In particular we have

$$
\varphi\left(L^{p_{0}}, L^{p_{1}}\right)=L_{\psi} .
$$

We note also that a simple calculation shows (see [11, p. 459]) that in the case $0<p_{0}=p_{1}=p \leq \infty$,

$$
\varphi\left(L_{w_{0}}^{p}, L_{w_{1}}^{p}\right)=L_{1 / \varphi\left(w_{0}, w_{1}\right)}^{p} .
$$

Our final result is the following:

Theorem 3.3. Let $L_{\psi}(\nu)$ and $L_{\psi_{i}}\left(\mu_{i}\right)$ for $1 \leq i \leq m$ be Orlicz spaces. Assume that there is a constant $C$ and $0<p_{0}<p_{1} \leq 1$ so that $\psi\left(t_{1} \cdots t_{m}\right) \geq C \psi_{1}\left(t_{1}\right) \cdots \psi_{m}\left(t_{m}\right)$ for all $t_{1}, \ldots, t_{m}>0$ and assume that the functions $t \mapsto \psi(t) / t^{p_{0}}, t \mapsto \psi_{i}(t) / t^{p_{0}}$ are non-decreasing and $t \mapsto \psi(t) / t^{p_{1}}, t \mapsto \psi_{i}(t) / t^{p_{1}}(1 \leq i \leq m)$ are non-increasing. Then for every

$$
T \in \mathcal{L}_{m}\left(\left(L^{p_{0}}\left(\mu_{1}\right), L^{p_{1}}\left(\mu_{1}\right)\right) \times \cdots \times\left(L^{p_{0}}\left(\mu_{m}\right), L^{p_{1}}\left(\mu_{m}\right)\right),\left(L^{p_{0}}(\nu), L^{p_{1}}(\nu)\right)\right)
$$

we have that

$$
T \in \mathcal{L}_{m}\left(L_{\psi_{1}}\left(\mu_{1}\right) \times \cdots \times L_{\psi_{m}}\left(\mu_{m}\right), L_{\psi}(\nu)\right) .
$$

Proof. First observe that if an Orlicz function $\phi$ is such that the function $t \mapsto$ $\phi(t) / t^{p_{0}}$ is non-decreasing and $t \mapsto \phi(t) / t^{p_{1}}$ is non-increasing, respectively, then the functions $t \mapsto \phi^{-1}(t) / t^{1 / p_{0}}$ and $t \mapsto \phi^{-1}(t) / t^{1 / p_{1}}$ are non-increasing and nondecreasing, respectively. This implies that the function $\rho:[0, \infty) \rightarrow[0, \infty)$ defined by $\rho(0)=0$ and

$$
\phi^{-1}(t)=t^{1 / p_{1}} \rho\left(t^{1 / p_{0}-1 / p_{1}}\right), \quad t>0,
$$

is quasi-concave; i.e., $t \mapsto \rho(t)$ is non-decreasing and $t \mapsto \rho(t) / t$ is non-increasing. 
Now define $\varphi \in \Phi$ by $\varphi(s, t)=t \rho(s / t)$ for $t>0$ and 0 if $t=0$. Then, we have

$$
\phi^{-1}(t) \asymp \varphi\left(t^{1 / p_{0}}, t^{1 / p_{1}}\right), \quad t \geq 0 .
$$

Thus, it follows by (4) that for any measure space $(\Omega, \nu)$ we have that

$$
L_{\phi}(\nu)=\varphi\left(L^{p_{0}}(\nu), L^{p_{1}}(\nu)\right),
$$

up to equivalence of quasi-norms.

Combining our hypotheses, we conclude that there exist $\varphi, \varphi_{i} \in \Phi$ such that

$$
\left.L_{\psi}(\nu)=\varphi\left(L^{p_{0}}(\nu), L^{p_{1}}(\nu)\right), \quad L_{\psi_{i}}\left(\mu_{i}\right)=\varphi_{i}\left(L^{p_{0}}\left(\mu_{i}\right)\right), L^{p_{1}}\left(\mu_{i}\right)\right), \quad 1 \leq i \leq m,
$$

with $\varphi\left(1, t_{1} \cdots t_{m}\right) \leq C \varphi_{1}\left(1, t_{1}\right) \cdots \varphi_{m}\left(1, t_{m}\right)$ for some $C>0$ and for all $t_{1}, \ldots, t_{m}$ $\geq 0$. An application of Theorem 3.2 completes the proof.

\section{REFERENCES}

[1] N. Aronszajn and E. Gagliardo, Interpolation spaces and interpolation methods, Ann. Mat. Pura Appl. (4) 68 (1965), 51-117. MR0226361 (37 \#1951)

[2] Colin Bennett and Robert Sharpley, Interpolation of operators, Pure and Applied Mathematics, vol. 129, Academic Press Inc., Boston, MA, 1988. MR.928802 (89e:46001)

[3] Yu. A. Brudnyı̌ and N. Ya. Krugljak, Interpolation functors and interpolation spaces. Vol. I, translated from the Russian by Natalie Wadhwa, with a preface by Jaak Peetre. NorthHolland Mathematical Library, vol. 47, North-Holland Publishing Co., Amsterdam, 1991. MR 1107298 (93b:46141)

[4] A.-P. Calderón, Intermediate spaces and interpolation, the complex method, Studia Math. 24 (1964), 113-190. MR0167830 (29 \#5097)

[5] Loukas Grafakos and Mieczysław Mastyło, Interpolation of bilinear operators between quasiBanach spaces, Positivity 10 (2006), no. 3, 409-429, DOI 10.1007/s11117-005-0034-x. MR2258951(2007h:46032)

[6] N. J. Kalton, N. T. Peck, and James W. Roberts, An F-space sampler, London Mathematical Society Lecture Note Series, vol. 89, Cambridge University Press, Cambridge, 1984. MR808777(87c:46002)

[7] G. Ja. Lozanovskiu, Certain Banach lattices. IV, Sibirsk. Mat. Z. 14 (1973), 140-155 (Russian); English transl., Siberian. Math. J. 14 (1973), 97-108. MR0336314 (49 \#1089)

[8] Mieczysław Mastyło, On interpolation of some quasi-Banach spaces, J. Math. Anal. Appl. 147 (1990), no. 2, 403-419, DOI 10.1016/0022-247X(90)90358-M. MR1050215 (91f:46106)

[9] Per Nilsson, Interpolation of Banach lattices, Studia Math. 82 (1985), no. 2, 135-154. MR.823972(87f:46137)

[10] V. I. Ovchinnikov, Interpolation in quasi-Banach Orlicz spaces (Russian), Funktsional. Anal. i Prilozhen. 16 (1982), no. 3, 78-79. MR674023 (84c:46029)

[11] V. I. Ovchinnikov, The method of orbits in interpolation theory, Math. Rep. 1 (1984), no. 2, $\mathrm{i}-\mathrm{x}$ and 349-515. MR877877 (88d:46136)

[12] E. M. Stein and G. Weiss, Interpolation of operators with change of measures, Trans. Amer. Math. Soc. 87 (1958), 159-172. MR0092943 (19,1184d)

[13] Dietmar Vogt, Integrationstheorie in p-normierten Räumen (German), Math. Ann. 173 (1967), 219-232. MR0217254(36 \#345)

Department of Mathematics, University of Missouri, Columbia, Missouri 65211

E-mail address: grafakosl@missouri.edu

Faculty of Mathematics and Computer Science, A. Mickiewicz University; And Institute of Mathematics, Polish Academy of Science (Poznań branch), Umultowska 87, 61-614 Poznań, POLAND

E-mail address: mastylo@math.amu.edu.pl

Faculty of Mathematics and Computer Science, A. Mickiewicz University, UmulTOWSKa 87, 61-614 Poznań, Poland

E-mail address: szwedek@amu.edu.pl 